\title{
Entre la síntesis activa y la síntesis pasiva: cuerpo, percepción y formación ${ }^{1}$ [T1]
}

\author{
Juan Manuel Carreño Cardozo ${ }^{2}$
}

\section{Resumen [TResumen]}

En este artículo se parte de la afirmación según la cual la pasividad (saber manejar bicicleta) se expresa en la actividad (manejar bicicleta) y que la actividad depende de lo sedimentado pasivamente (no es posible manejar bicicleta sin saberla manejar), por ello, la pregunta que orienta este escrito es: ¿cómo se vincula la actividad con la pasividad? De esta relación se quiere cuestionar: ¿cómo se dirigen aspectos de la actividad a la pasividad? ¿Es la percepción (o la sensación) el límite de la relación entre actividad y pasividad? ¿Cómo se relacionan la voluntad y la sensibilidad con la actividad como condición de la formación? Para describir estas relaciones se presenta: 1) una comprensión del cuerpo en Merleau-Ponty, a partir de la cual la percepción es el lugar de convergencia entre actividad y pasividad. 2) Las interacciones entre pasividad y actividad. Y 3) la posible relación entre formación y síntesis activa o, de otro modo, entre voluntad y sensibilidad en la formación.

Palabras clave: cuerpo, percepción, fenomenología, formación, actividad y pasividad.

\footnotetext{
${ }^{1}$ Escrito producto del proyecto de investigación FEF-440-16, "Cuerpo y disfrute en la fenomenología de Husserl", vigencia 2016, financiado por el Centro de Investigaciones de la Universidad Pedagógica Nacional.

2 Estudiante del Doctorado en Educación (Universidad Pedagógica Nacional). Docente de planta, Universidad Pedagógica Nacional. Correo electrónico: jcarreno@pedagogica.edu.co
} 


\section{El cuerpo y la percepción [T2]}

Comprender los procesos de síntesis activa y pasiva sin comprender el cuerpo como órgano perceptor y percibido de y con el mundo, puede llevar al juicio superficial de entenderlos como actos objetivos-lógicos, polarizados y sobrepuestos. De esta forma, se iguala la pasividad a determinación, objetividad, verdad, universalidad; y actividad a voluntad, atención, indeterminación, subjetividad, relatividad y particularidad. Esta pareciera ser la continuación de dualismos que la fenomenología ha enfrentado. Merleau-Ponty propone una continuación del estudio de Husserl sobre el cuerpo y llega a delimitar la percepción como eje por el cual se constituye el conocimiento del mundo. Ello en cuanto cuerpo es objeto, órgano de la consciencia. Para comprender esta relación entre la síntesis pasiva y la síntesis activa se revisan los postulados de Husserl y los desarrollos de Merleau-Ponty con respecto al cuerpo y la experiencia perceptiva.

Para Husserl, cuerpo es la extensión del cuerpo. En principio toda cosa de la naturaleza tiene extensión, fijada en un tiempo objetivo. Le corresponde por ello una duración y una nota real que no se altera. Su extensión le hace móvil. El modo de extensión del cuerpo material es distinto de la cosa animal. En la segunda no se trata solo de la extensión. La naturaleza animal es espiritual (con extensión y algo que no es extensión) (Husserl, 2014, párr. 13, p. 58). Ahora, la cosa está referida a su extensión, tiene corporeidad espacial. Esta extensión no es fragmento espacial, sino cuerpo. Fragmentación de la extensión de la cosa es fragmentación de la cosa. Es en sí misma su extensión (su cuerpo). Cuerpo es un llenado de su extensión. Las cualidades de este cuerpo son variables correspondientes a su nota esencial. La extensión corpórea es una forma esencial de sus propiedades reales (Husserl, 2014, párr. 13, p. 60). Sabemos que cosas, animales y hombres tienen cuerpos materiales con extensión espacial. Sin embargo, hombres y animales no son solo la extensión, ya que no son fragmentables por ello. Son localizables.

La extensión implica sustancia. Husserl sugiere analizar fenomenológicamente desde la percepción como la operación que permite determinar la dación de cosas, que junto con la presunción de las cosas dan como resultado la idea de cosa. La aprehensión percibida no implica la representación de la esencia de las cosas. La esencia comprende un esquema sensible, armazón llenado con la extensión de la cosa. Cuerpo implica la cualificación percibida, pero el fantasma también es, sin necesidad de los componentes de la materialidad. Lo percibido sensiblemente es 
una parte, pero las cosas no son solo lo percibido, sino lo imaginado. (Husserl, 2014, párr. 18, p. 90).

Para Merleau-Ponty, fenomenología describe la experiencia del cuerpo desde su facticidad. Para esto, retomando la propuesta husserliana de volver a las cosas mismas, comprende un mundo que existe, y que, precisamente, está antes de las determinaciones científicas (abstractas). El mundo es "medio natural y el campo de todos mis pensamientos y de todas mis percepciones explícitas" (Merleau-Ponty, 1945, p. 10). A la vez, el mundo es lo que yo percibo de él. Así, fenomenología no es objetividad (realismo) ni subjetividad (idealismo) absolutas: el estudio del fenómeno implica un puente que privilegia lo fáctico y describe los modos de aparecencia para el sujeto. Percepción, en este sentido, es el momento de constitución del mundo que es fáctico. Se juega la percepción en una experiencia actual, vívida en el mundo, como Merleau-Ponty recupera de Heidegger: una experiencia de ser-en-el-mundo. El mundo que analiza la fenomenología no es el mundo que está ahí, indistinto del sujeto, sino el mundo que está ahí existente de un modo para su constitución en la consciencia del sujeto. Desde este cuerpo, órgano de la percepción, la actividad se manifiesta como presencia (no necesariamente consciencia) del cuerpo (yo mismo) en un presente y un habitar concreto en el mundo. Afirma Husserl:

El cuerpo es, ante todo, el medio de toda percepción; es el órgano de la percepción; concurre necesariamente en toda percepción.t En el ver, el ojo está dirigido a lo visto y pasa corriendo sobre las esquinas, las superficies, etc. Al palpar, la mano se desliza sobre los objetos. Moviéndome, acerco el oído para escuchar. La aprehensión perceptiva presupone contenidos de sensación que desempeñan su necesario papel para la constitución de los esquemas y por ende de las apariciones de las cosas reales mismas. (2014, párr. 18, p. 88).

De esta forma, cuerpo concurre a toda percepción y la percepción es lo que concurre entre cuerpo y objetos. Desarrollando las ideas de Husserl, para Merleau-Ponty, la percepción necesita un marco que lo diferencie de otras nociones, sobre todo para, en ejercicio fenomenológico, distanciar los prejuicios que no permitan el análisis sobre la constitución del mundo percibido. En especial, este autor refiere los prejuicios construidos por el empirismo y el intelectualismo. Sobre el primero, más que negarlo, le actualiza en formas que faciliten la observación del hecho fáctico, descartando la orientación de construcción de leyes universales. El segundo, afirmando la 
importancia de la filosofía trascendental, que vista fenomenológicamente es descripción de los actos de consciencia (la constitución).

Sensación, asociación, atención y juicio son las nociones que, por su prejuicio, no deben confundirse con percepción. En especial, sensación no es acto anterior a la percepción ni cualidades anteriores a la percepción o la tematización. La sensación solo se conoce por un idealismo del sentir, el ver, el tocar. De forma similar, la percepción no es suma de sensaciones evocadas que se remiten a actuales: en el fenómeno mismo se percibe un conjunto con unidad de sentido. En estas nociones se comprende un mundo que no es sin cuerpo (o cuerpos) y cuerpo que no es sin constituir mundo (aparte que el cuerpo es también objeto en el mundo).

Merleau-Ponty considera que el estudio del cuerpo debe superar su objetivismo. Igualmente, ningún objeto puede ser visto desde todos sus lados, alejado o rodeado para constituirse como lo que es. Siempre los objetos se dan en perspectivas que no se agotan. Los objetos, entonces, siempre son ideas del objeto. El cuerpo siempre es idea del cuerpo. Sin embargo, su constitución es fenomenológicamente vista como lo habitado. Hay consciencia de sí que no es distinta de la consciencia de cuerpo. Entonces, la realidad del cuerpo vista desde la fisiología (la clásica al menos) que muestra redes y conexiones eléctricas entre un sistema nervioso periférico y central, muestra una realidad que parece más válida, pero que no se acerca a la realidad vivida existente para la consciencia_- El autor, a través de los ejemplos de alteraciones orgánicas (el miembro fantasma para este caso), demuestra posibles invalidaciones de esta verdad planteada por el empirismo.

El cuerpo para Merleau-Ponty no es objeto ya que está permanentemente conmigo, está siempre bajo el mismo ángulo, más exactamente soy cuerpo. Este es "medio" de relación con el mundo, pero no un instrumento, sino el yo mismo que percibe, por eso "tener" cuerpo solo se da en el lenguaje como un hábito lingüístico: puedo decir que tengo manos, pero jamás afirmo que mi mano toca algo, sino que soy yo el que toco. Similar ejemplo cita Merleau-Ponty del dolor en el pie (cfr. Merleau-Ponty, 1945, p. 111). Ahora, el cuerpo lo delimito por fronteras que hacen un solo conjunto yo. A este conjunto el autor lo llama esqueleto corpóreo. Sin embargo, la consciencia de la ubicación no ocurre en simultáneo de cada punto de mi cuerpo percibido: ocurre una carga de consciencia de ese esquema corpóreo por una espacialidad de situación, en la que mi consciencia se acentúa sin perder el horizonte de posición, magnitud o situación. Con 
esto demuestra Merleau-Ponty que "no es nunca nuestro cuerpo objetivo lo que movemos, sino nuestro cuerpo fenomenal" (Merleau-Ponty, 1945, p. 123). Además, el movimiento, integrado como acto en la motricidad, es muestra del yo puedo que dirige la consciencia (cfr. MerleauPonty, 1945, p. 156-157). Este yo puedo no solamente expresa coordinación entre partes y funciones, sino un acto/posibilidad de síntesis de sí mismo (del cuerpo). Esta síntesis no ocurre por consecuencia de leyes, sino por actos conjuntos en el que la consciencia se dirige.

Se tiene, entonces, un cuerpo que no es objeto, aunque es algo en el mundo, que es con todas sus acciones y capacidad de síntesis, el órgano de relación (percepción-acción) del sí (la consciencia) con el mundo. La percepción, o mejor, la experiencia perceptiva, es el conjunto de relaciones (psicológicas, físicas, afectivas y cognoscitivas) que existen y forman un tejido cuya intención es la comunicación del sí con el mundo. El cuerpo es órgano de la percepción en cuanto es conjunto que hace unidad la multiplicidad de sensaciones, datos, posiciones, etc.:

Por eso es verdad decir que la cosa se constituye en un flujo de apariencias subjetivas. Y sin embargo, yo no la constituía actualmente; eso es, yo no pro-ponía, activamente y por medio de una inspección del espíritu, las relaciones de todos los perfiles sensoriales entre ellos y mis aparatos sensoriales. Es lo que expresamos al decir que percibo con mi cuerpo. (Merleau-Ponty, 1945, p. 340).

Entonces, la percepción, para Merleau-Ponty, es el flujo de las aparecencias hecho unidad desde sí al mundo y de sí hacia el mundo. De varias formas, la percepción es la constitución de la unidad a través del mismo cuerpo. Sería una característica específica de esta unidad cuerpo que propone Husserl: "El cuerpo es una unidad de la experiencia, y en el sentido de esta unidad radica el ser índice para una multiplicidad de experiencias posibles en las cuales el cuerpo puede venir a darse en formas siempre nuevas" (Husserl, 2014, párr. 15, p. 70). Esta afirmación del cuerpo como unidad trasciende las sensaciones y las experiencias. Husserl presenta el cuerpo como lugar de lo material y lo espiritual:

La experiencia enseña que la espiritualidad real está enlazada con cuerpos materiales y no acaso con meros fantasmas espaciales subjetivos o intersubjetivos (puros esquemas espaciales), y siguiendo esa experiencia, para nosotros cuerpo material y alma se conciertan necesariamente en la idea de un hombre real. (Husserl, 2014, párr. 21, p. 130). 
Este aspecto es relevante para diferenciar definitivamente el cuerpo de las cosas: es cosa animada, cuyo contenido expresa y percibe con respecto a esa unidad material-espiritual. Parte de esta no objetualidad es descrita por Husserl con las ubiestesias, como característica especial que organiza las sensaciones por la ubicación respecto a su esqueleto corpóreo. Los rasgos de este objeto cuerpo están dispuestos por esa unidad ya que "el cuerpo no es solamente en general una cosa, sino expresión del espíritu, y es a la vez órgano del espíritu” (Husserl, 2014, párr. 21, p. 131).

En el ejemplo inicial (el de montar bicicleta) tenemos a este punto: un objeto cuerpo (cosa material animal cuerpo) que maneja otro objeto bicicleta (mera cosa material). Cuerpo (material y espiritual) animado, autónomo, que percibe (no siente) la bicicleta (dirigida, manipulada). El cuerpo hace unidad lo fisiológico — para mover la máquina — y la espiritualidad — yo puedoque le motiva moverla. A su vez, la percepción hace unidad toda información sensible de peso, color, textura y también datos de posición corporal, tensiones musculares, ubicaciones espaciales de brazos, piernas, tórax, etc. en el acto de manejar bicicleta que debe constituir el objeto bicicleta y las acciones de relación del cuerpo con la bicicleta. Ahora, comprendiendo el cuerpo como órgano de la percepción y esta como unidad de las experiencias (sensación-expresión), es necesario ampliar la relación que puede existir entre pasividad y actividad a partir de la percepción.

\section{Pasividad y actividad [T2]}

Según Merleau-Ponty: "La unión del alma y del cuerpo no viene sellada por un decreto arbitrario entre dos términos exteriores: uno, el objeto, el otro, el sujeto. Esta unión se consuma a cada instante en el movimiento de la existencia" (Merleau-Ponty, 1945, p. 107). Así, sin intentar superar el dualismo cartesiano, el autor describe en la existencia, la vivencia unificada del ser (como cuerpo). Esta existencia se sintetiza en la corriente de experiencias, que no es más que el fenómeno descrito antes como "experiencia perceptiva". Cuerpo y espíritu no son realidades, sino expresiones de relación de la consciencia con el mundo. De esta forma, pasividad y actividad no deben representar una nueva concepción de objeto-sujeto. Por el contrario, son el replanteamiento de vínculos fenomenales en la constitución del mundo por parte del sujeto. De esta forma, el cuerpo tiene una dimensión pasiva. Para el autor: 
En cuanto es portadora de "órganos de los sentidos", la existencia corpórea no se apoya jamás en sí misma, siempre está trabajada por un no-ser activo, continuamente me hace la proposición de vivir, y el tiempo natural, en cada instante que llega, dibuja sin cesar la forma vacía del verdadero acontecimiento. (Merleau-Ponty, 1945, p. 182).

Para Merleau-Ponty, hablando del juicio según Descartes: “el 'juicio natural' no es otra cosa que el fenómeno de la pasividad. Siempre pertenecerá a la percepción el conocer la percepción” (Merleau-Ponty, 1945, p. 64). Esto indica, por un lado, la propuesta de pasividad como inscrita en el hecho, lo existente. Por otro lado, describe que el juicio natural sobre la percepción es la percepción misma. La reflexión sobre la percepción no cambia el hecho mismo de la percepción. Igualmente, ejemplifica Merleau-Ponty de la caricia, la cual ya está comprendida corporalmente “antes de que el filósofo defina su significado intelectual” (1945, p. 203). Pasividad, así vista, es todo hecho existente en el mundo, constituido por el sujeto como hecho ya existente. Además, cuando el autor menciona que "con el problema del sentir redescubrimos el de la asociación y la pasividad" (Merleau-Ponty, 1945, p. 73) indica que el sentir (no como quale puro) se orienta por asociación entre pasividad y espontaneidad, vínculo que no ocurre solamente por actividad. Entonces, Merleau-Ponty presenta ya una relación permanente entre actividad y pasividad en un plano de la revaluación de prejuicios sobre el proceso de la percepción. Esta no ocurre por momentos o micromomentos secuenciales ni tienen una dirección de estímulos eléctricos (como lo dice el empirismo). Todo este prejuicio, adicionalmente, centra la percepción como en situación o acto interno del sujeto. Fenomenológicamente, la percepción es correlato del sí y el mundo. Por esta razón afirma Merleau-Ponty:

Una síntesis pasiva es contradictoria si la síntesis es composición, y si la pasividad consiste en recibir una multiplicidad en lugar de componerla. Queríamos decir, al hablar de síntesis pasiva, que lo múltiple lo penetramos nosotros y que, no obstante, no somos nosotros quienes efectuamos su síntesis. (Merleau-Ponty, 1945, p. 434).

De este modo, la síntesis pasiva ocurre con el sujeto, pero dependiente de un tiempo que no es decisivo del sujeto. De alguna forma se hace interdependiente, como acto universalizante de síntesis que supera las actividades perceptivas de cada sujeto. Pasividad es constitutiva, pero no igual que las síntesis de la percepción activa, se trata de una forma de constitución que antecede, como un escenario en el que ya habita la consciencia: 
Lo que se llama la pasividad no es la recepción por parte nuestra de una realidad ajena o la acción causal del exterior en nosotros: es un investir, un ser en situación, ante el cual no existimos, que perpetuamente recomenzamos y que es constitutivo de nosotros mismos. (Merleau-Ponty, 1945, p. 435).

El fenómeno estudiado, que equilibra este escenario de pasividad y actividad, se presenta como una sola vivencia en la que conviven en tiempo presente. Esta relación paralela, simultánea, permite afirmar al autor francés que "no somos, de una manera incomprensible, una actividad unida a una pasividad, un automatismo más una voluntad, una percepción más un juicio, sino del todo activos y del todo pasivos, porque somos el surgir del tiempo" (1945, p. 435). En el ejemplo del autor, el sentir permite trasladar lo pasivo y lo activo de formas complejas: "Cuando toco mi mano derecha con mi mano izquierda, el objeto mano derecha tiene esta singular propiedad de también sentir. Acabamos de ver que nunca ambas manos son al mismo tiempo, una respecto de la otra, tocadas y tocantes" (Merleau-Ponty, 1945, p. 110). Mi mano tocante es activa, mi mano tocada es pasiva en cuanto "idea" de mano tocada que le permite poder ser mano tocada. Si fijo mi atención en la anterior mano tocada, se activa mi mano tocante. Sin embargo, la actividad que fluye en la alternación de mi atención no es exactamente alternación entre pasividad y actividad, ya que la idea (o conjunto de sentires, representaciones, ideas) de mano tocada, permanece como "fuera de" las mismas manos, aunque su "idea" se orienta a las manos.

En cuanto pasividad es, como conjunción de lo universal y lo particular, la actividad implica cada acción que intenciona percepción de algo. No es la atención, ya que, como afirma el autor:

La atención no es ni una asociación de imágenes, ni un retorno hacia sí de un pensamiento que ya es maestro de sus objetos, sino la constitución activa de un objeto nuevo que explicita y tematiza lo que hasta entonces solamente se ofrecía a título de horizonte indeterminado. (Merleau-Ponty, 1945, p. 52).

Entonces, la atención es dirección de voluntad. Sin embargo, lo no dirigido por la voluntad también se percibe, como el fondo de la figura, el marco visual que no es el objeto central de la visión.

Modos de la sintesis activa [T3] 
Para comprender los procesos de síntesis activa, es pertinente describir la constitución general del mundo y las cosas. Para Husserl, el mundo y las cosas son condiciones de constitución (Husserl, 2014, párr. 18, 87-122). Hace diferencia entre la constitución de la cosa, la cosa material objetiva y la constitución de mundo - sensible y verdadero-. Husserl investiga todas las cosas como posibles cosas materiales objetivas, hecho que al final del capítulo tercero define irreal dada la condición de diversidad (no somos idénticos y somos inteligentes libres) lo que lleva a plantear su idea de entrada a la diferencia de las cosas materiales con las animales. Ahora, la constitución del mundo se diferencia de la cosa objetiva en cuanto el primero pueda comprenderse como el conjunto de cosas o realidades en un espacio determinado como mundo. En este sentido, se comprende la interacción del cuerpo con su mundo, el cual es, en principio, de aparición relativa dada la condición psicofísica del sujeto, aunque se determina siempre como un mundo uno, normalmente constituido.

En la constitución de las cosas materiales presenta niveles de constitución, entre los cuales están la constitución en la espacialidad, de las sensaciones (aclarando que las condiciones psicofísicas no aportan a la constitución de la cosa) y de las experiencias (percepciones rectificadoras). Estos funcionan como operación del estrato inferior, a lo cual se añade la constitución intuitiva o predada. Husserl profundiza en la constitución de la normalidad desde la dación óptima. En esta convergen las notas ideales de la cosa y la normalidad de las condiciones psicofísicas o la conciencia de alteración (tanto causal como psicofísica).

Así, se comprende inicialmente la constitución desde dos grandes niveles (gráfico 1).

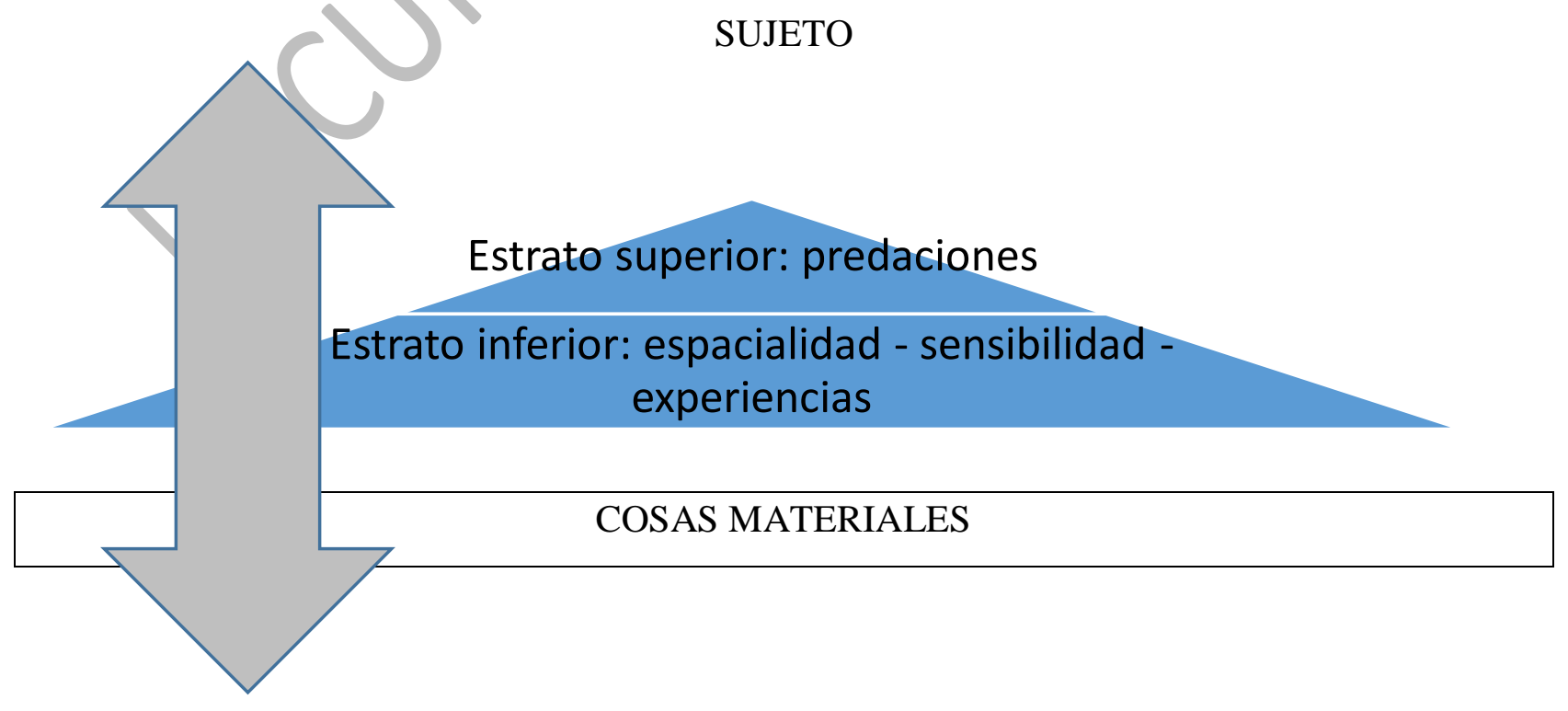




\section{MUNDO}

\section{Gráfico 1. Los dos niveles generales de la constitución}

Fuente: Elaboración propia.

Afirma Vargas (2016) que la cosa siempre aparece por escorzos, es decir, es imposible percibir el todo de una cosa, solo se tienen los retazos. Las formas de hacer de ellos cosa es, según Husserl, un proceso en el que se involucra lo anterior a los sentidos (predado), lo sentido (percibido $y$ valiceptido) y lo posterior (corregido o corroborado o predicado). En toda esta dinámica se comprende el ejercicio de síntesis como apercepción. Ahora, esta constitución aperceptiva funciona por efecto de la motivación. Es decir, no es por lógica ni azar que ocurre una forma ordenada de juntar los escorzos. Es por motivación. Este es un término que usa Husserl para definir lugares de las aprehensiones necesarias para la constitución de la idea de cosa. Define la existencia de aprehensiones: 1. Motivantes, como aquellas originadas en sensaciones cinestésicas, y 2. Motivadas, las sensaciones de las notas de las cosas. Además, sugiere que las motivaciones se dan en ordenaciones y que estas series originan la experiencia. Se puede comprender la motivación como una orientación de la intención. Una aprehensión de la cosa desde una referencia intencional. Adiciona Husserl que las motivaciones tienen que ver con la normalidad, en cuanto hay aprehensiones que ayudan a la referencia concordante con el sistema de percepción motivadas (Husserl, 2014, párr. 18, p. 94).

Se tiene, entonces, hasta este punto de la revisión, además de los niveles amplios (estrato inferior y superior) la descripción de la percepción y la motivación como un nivel de interacción entre el inferior y el superior. A continuación, se presentan dos formas de actividad descritas por Husserl, y que se pueden comprender como modos de la síntesis activa: la ubicación y la actividad teórica.

\section{La ubicación como actividad [T4]}

La ubicación espacial es actividad, anterior y requisito de estas. El cuerpo sin otra condición, se ubica en un tiempo presente y sus relaciones con el mundo comprenden todo desde su ubicación. Esta actividad se caracteriza por modos de conciencia de estar:

La palabra "aquí", aplicada a mi cuerpo, no designa una posición determinada con respecto a otras posiciones o con respecto a unas coordenadas exteriores, sino la 
instalación de las primeras coordenadas, el anclaje del cuerpo activo en un objeto, la situación del cuerpo ante sus tareas. (Merleau-Ponty, 1945, p. 117).

Para Husserl, la ubicación también distingue la imposibilidad de la fragmentación de hombres y animales (Husserl, 2014, párr. 14, p. 63). Estos no se pueden dividir sino localizar. Y se ubican como un todo material, anímico y espiritual dada su condición corporal. Entonces, esta actividad refiere tanto la orientación de estar como un todo, como la capacidad de localización para actuar. Esta capacidad es tanto la localización entre el sistema corporal (esquema corporal) como la de este punto con respecto a las otras cosas.

En la relación intersubjetiva, la localización permite una nueva ubicación de ser y propiedades en una relación de empatía:

En mi mundo circundante físico encuentro por ende cuerpos, es decir, cosas materiales del tipo de la cosa material "mi cuerpo" constituida en la experiencia solipsista, y los aprehendo como cuerpos, es decir, empatizo en ellos en cada caso un sujeto-yo con todo lo que le pertenece y con el contenido particular que cada caso exige. Con ello se transfiere a los cuerpos ajenos ante todo aquella "localización" que ejecuto en los diferentes campos sensoriales (campo táctil, calor, frío, olor, sabor, dolor, placer sensible) y ámbitos sensoriales (sensaciones de movimiento), e igualmente mi localización indirecta de actividades espirituales. (Husserl, 2014, párr. 164).

La ubicación en este sentido es actividad de reconocimiento de mis posibilidades en el otro cuerpo y transferencia de localizaciones particulares. En resumen, ubicación es actividad inicial sobre la cual es posible la actuación entre sí mismo (localización intra-), con el mundo (localización con) y los otros (transferencia a otros cuerpos).

\section{La actividad teórica [T4]}

Para Husserl, la actitud teórica, diferente a la valorativa y práctica, consiste en un giro de la mirada teórica, y superar lo dóxico para llegar a un ver percatándose, mirada activa que además tiene intención de predicar lo visto. La actitud teórica es el yo dirigido al objeto objetivando. Por otro lado, este giro teórico ocurre desde las vivencias intencionales, estas constituyen objetos categoriales. Las vivencias, siempre que no procedan de actos teóricos, pueden ser actos 
predadores como ocurrencias recordativas o nuevas. Todo acto puede ser teórico por cambio de actitud, comienza por intuición sensible, una captación simple del objeto y luego la captación del valor. La predicación da el giro dóxico que sigue a la mirada sensible primera. Luego, el acto teórico se ejecuta cuando se capta decisión y acción.

El sujeto puede vivir, y usualmente lo hace, en la ejecución misma del acto (en pasividad) o puede pasar a una actitud teórica (en actividad), donde lo objetivo se vuelve objeto teórico. En todo caso, en la ejecución hay dignidades dominantes y servidoras de la experiencia. Cada una mantiene su tema, sea teórico o práctico, aunque varíen su lugar. Añade Husserl una observación: la diferencia entre la reflexión y la actitud teórica es que la primera puede corresponder a todos los tipos de actitud, no hay predicados objetivos, sino predicados relativos a la conciencia.

Todo acto puede ser objetivante, aun en lo sensible es posible llevar al dominio teórico las cualidades y valoraciones. En el proceso de objetivación se constituyen objetos que no remiten a objetos predados, Husserl los llama protoobjetos Husserl, 2014, párr. 8, p. 46) que en últimas son objetos de sentido, fundantes. Además, la misma diferencia entre la actitud teórica y la práctica, ocurre en la operación de síntesis: entre la categorial y la estética o sensible. Por último, en esta operación objetivante, hay datos de sensación, que actúan como objetos antes de ser objetos (algo sin aprehensión objetiva).

En síntesis, se tiene que los dos niveles de constitución, planteados anteriormente, tienen relaciones e interacciones entre sí, que vinculan la percepción y la motivación como índices de enlace entre estratos. Además, una interacción entre actividad y pasividad en la que se distingue la actividad de la ubicación como cercana a los estratos inferiores, luego las actividades dóxicas y teóricas que tienden a una condición reflexiva en la que el sujeto piensa sobre sí y para ello recurre a lo sedimentado en constitución pasiva. Por último, se postula la actividad predicativa como aquella cercana a esta constitución pasiva y que representa un nivel superior de actividad (gráfico 2).

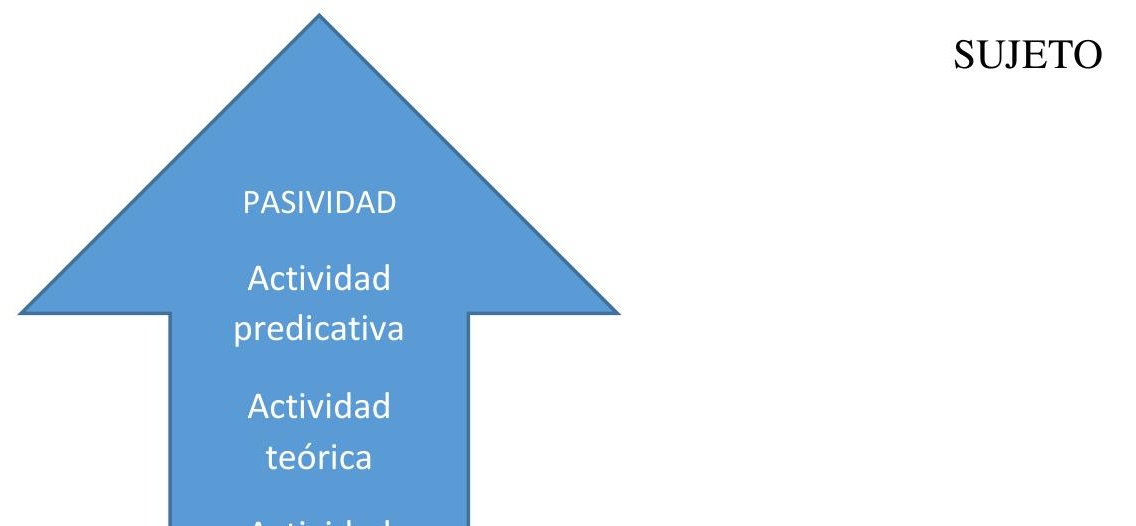




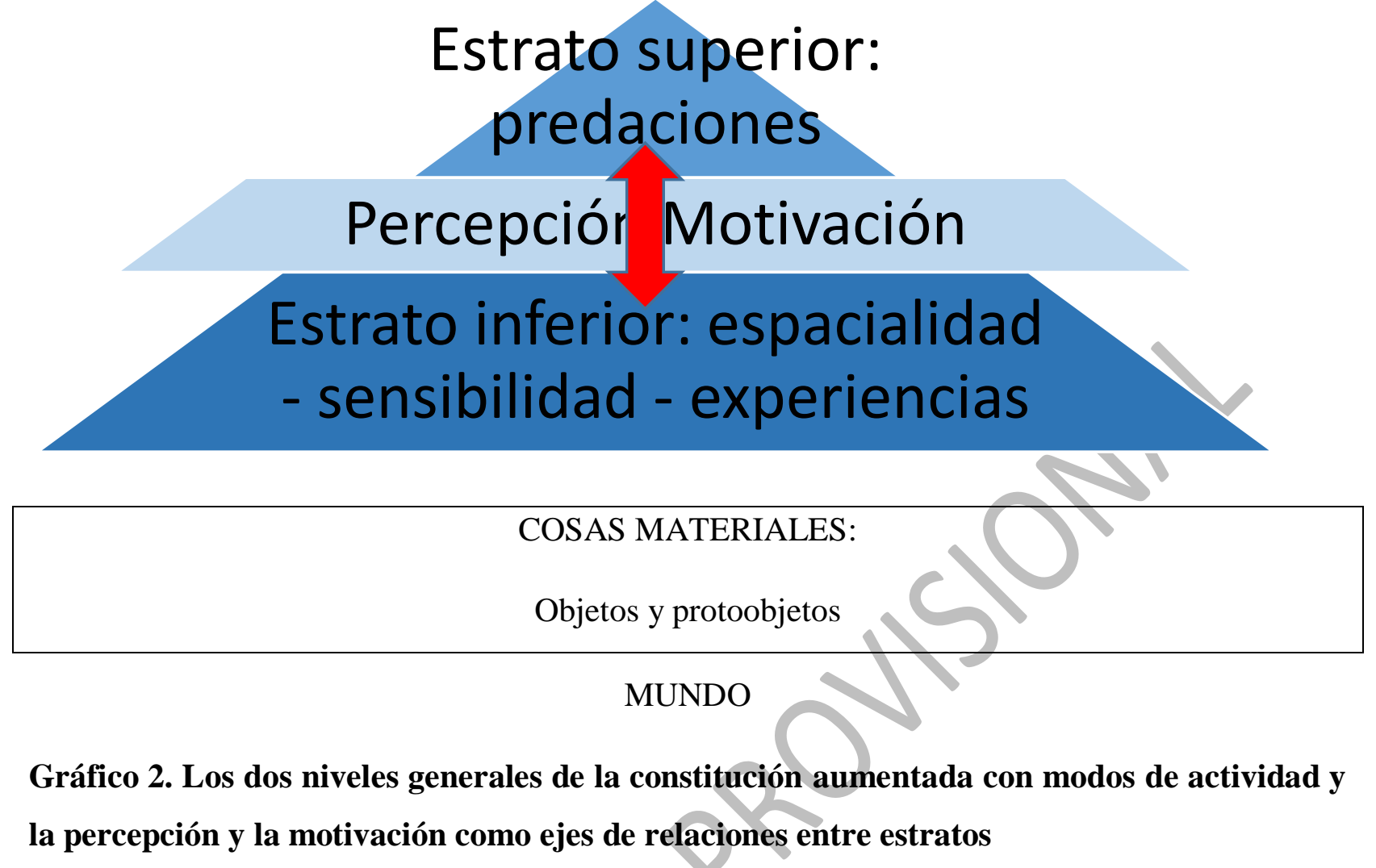

Fuente: Elaboración propia.

De la síntesis activa a la pasiva [T3]

Husserl también denomina espontaneidad a la actividad. Se entiende que una actividad es ejecución, y esta es más "activa" cuando se acerca más a esta ejecución sin causa aparente. Hay movimiento de ejecución con orientación voluntaria. Sin embargo, actividad también se da sin esta voluntad explícita o directa. Se puede situar, entonces, una serie de actividades con mayor o menor cercanía a la pasividad. A partir de este acercamiento, se iniciará la relación entre la síntesis pasiva y activa:

[L]a espontaneidad, o, si se quiere, la que propiamente denominamos actividad, pasa a pasividad, aunque una pasividad que — como ya dijimos - remite a la ejecución primigeniamente espontánea y articulada. Esta remisión se caracteriza como tal mediante el yo puedo o la capacidad, evidentemente inherente, de "reactivar" este estado, esto es, de trasladarlo a la elaboración, que se hace consciente como "repetición", de aquella producción de la cual antes había provenido y en la cual 
finalmente proviene "de nuevo" como el mismo estado y hace que provenga en sí el mismo resultado en cuanto el mismo sentido final y con la misma validez. (Husserl, 2014, párr. 12, p. 41).

Así, entre actividad y pasividad está la capacidad (el yo puedo) como lo remitido de la actividad y respuesta de la pasividad. Como remisión se comprende el yo puedo como ejecución que toca en pasividad, la capacidad para ejecutar una acción. En cuanto respuesta de lo pasivo, es la posibilidad de repetición que desencadena desde la pasividad las actividades del yo puedo.

Como se observa, entre la síntesis pasiva y la activa hay más relaciones que diferenciación. Las dos son constituyentes permanentes de las cosas y el mundo. Además, son interdependientes, es decir, continuamente se requieren de síntesis activas y pasivas en la constitución del mundo. Justamente, es esta relación continua la que permite la actuación real del sujeto en el mundo. Para Husserl, el cuerpo cosa humano (que es cosa particular) manifiesta del alma una personalidad y esta, junto con sus condiciones, permite que actúe libremente móvil por su voluntad. Esta actuación libre es actividad, que implica necesariamente movilidad. En el movimiento corporal también se conjuga actividad y pasividad, y a la vez es unidad entre personalidad y ánima (ya que concurren en el cuerpo). Husserl resalta que en el cuerpo, sensibilidad y localización son manifestaciones de propiedades materiales y anímicas. Con ello se comprenden las ubiestesias extensión del alma en la cosa material - (Husserl, 2014, párr. 38, p. 190) como característica que refiere a propiedades del alma. Esta propiedad es capacidad de sensación y localización, referidas al cuerpo móvil órgano de la voluntad: único objeto movible por mi voluntad y que puede mover otras cosas por condición del yo libre. El movimiento es comportamiento hacia el mundo y en estas actividades corresponden los movimientos familiares y los más “automatizados". Según Husserl:

Para el yo las cosas están constituidas, pero a una con ellas se constituye el yo de cierta manera empíricamente familiar (por ende, en una especie determinada de apercepción), comportándose hacia las cosas que aparecen. Ejecuta actividades de percepción, y a ellas pertenecen "actividades" esencialmente corporales. Considerando las cosas aparentes, dirige de diversas maneras sus órganos sensoriales; mueve los ojos, lleva a cabo una acomodación cambiante, palpa con las manos las superficies corpoicas aparentes, etcétera. (2014, párr. 310, pp. 360-361). 
Apercepción, desde esta afirmación, parece tener más relaciones con la síntesis pasiva, mientras la percepción tiende a relacionarse con los procesos activos. Con respecto a la apercepción en la síntesis pasiva, la actividad del yo puro no es ejecución que le puede mutar. Más bien es posible su presencia y ausencia. Esto es modo de actividad propia del yo puro, mientras que el yo anímico y real siempre tienen afectación por su actividad. Husserl afirma:

Podemos ver con intelección evidente en qué sentido el yo puro se muda en el mudamiento de sus actos. Es mudable en sus actuaciones; en sus actividades y pasividades, en su estar atraído y estar repelido, etc. Pero estos mudamientos no lo mudan a él mismo. Más bien él es en sí inmutable. No es un algo idéntico que tenga que manifestarse y verificarse primero en múltiples estados de propiedades permanentes determinados por circunstancias cambiantes. (Husserl, 2014, párr. 24, p. 140).

Este yo puro, que no mora en la corporalidad, es individual absoluto, es también yo libre, puede asumirse como el yo, que yo mismo capto. Además, dice Husserl que es característicamente yo voluntario (sujeto volitivo) y se comporta experimentando impulsos de los objetos, y yendo de un objeto a otro. A diferencia de la constitución de cosas materiales, este yo puro es el mismo yo captado. En la captación del yo puro cogito, lo captado son ya unidades profundas inmanentes, es decir, objetos últimos de las captaciones posibles, por eso mismo no dependen de un flujo temporal, sino que se dan en el flujo de vivencias. Por esto, Husserl dice que solo se ausenta y se presenta. Además, el yo puro es inmutable, no depende de lo experimentable, es y no deja de ser. Con esto, Husserl refiere al yo puro como un captador de cosas que no se van y no se modifican.

En el yo anímico y real la actividad depende de la experiencia. Según Husserl, esta actividad es el yo puedo en favor de vencer una resistencia. Así, actividad sería pasividad con capacidad de acción:

En la experiencia se distingue, según su carácter fenomenológico, el "yo puedo" y el "yo no puedo". Hay un hacer sin resistencia, o una conciencia del poder sin resistencia, y un hacer en la superación de una resistencia, un hacer con un "contra" y una conciencia inherente del poder que supera la resistencia. Hay (siempre fenomenológicamente) una gradualidad de la resistencia y de la fuerza de superación: de la fuerza "activa" frente a la "inerte" de la resistencia. [...] La apercepción propia 
de la resistencia presupone que no se trata meramente de algo cósico, sino de algo de la índole de lo que cae en la esfera de mi "voluntad", en la esfera de aquello que eventualmente ya he llegado a conocer como algo de lo que soy capaz. Todo mi poder en la esfera física está mediado por mi “actividad corporal”, por mi poder o ser capaz corporal. (Husserl, 2014, párr. 59, p. 259).

El cuerpo en actividad expresa la capacidad total, dispuesta por voluntad, emanada posiblemente en pasividad. Esta capacidad, este yo puedo, se mide con respecto a las series de relaciones, daciones y experiencias que constituyen mundo. Así, la actividad es regulada, aunque también arriesga de acuerdo con su posibilidad. Como ya se expresó, además la actividad implica movimiento, entonces, el yo puedo no queda en abstracción, es siempre movimiento del cuerpo que se dirige voluntariamente ${ }^{3}$.

Para finalizar esta sección, se retoma el ejemplo de la bicicleta: la actividad está expresada en el acto de montar bicicleta y efectivamente hacerlo, impulsada esta acción por lo dado en pasividad: saber montar bicicleta. Este saber (pasividad) solo se expresa en el acto de montar bicicleta (actividad) por 1. Motivación: algo que origina, intenciona desplazarse en bicicleta, sumado a familiaridades y espontaneidades que llevan a montar la bicicleta. 2. Percepción: hay relaciones fundadas en la predación, como la bicicleta (la idea de la bicicleta que es apercepción), vinculada a circunstancias como las características del día y la localización de mi cuerpo. Sobre estas relaciones el sujeto constituye el yo puedo montar bicicleta al medir las fuerzas, comparar experiencias, proyectar su beneficio y con ello orientar su voluntad al acto de montar bicicleta y a hacerlo por razones más específicas. En esta constitución ya hay actividad del yo puro en la idea de montar bicicleta que no puede ser modificada por la experiencia del sujeto, y hay suceso efectivo, particular y personal de montar en bicicleta. Este acto ocurre por la acción de saberes y valores sedimentados en pasividad que en la actividad se requieren o se repiten.

\section{Formación y síntesis activa [T2]}

\footnotetext{
${ }^{3}$ Hay que aclarar que esta voluntad no se refiere necesariamente a una dirección explícita o predicada de esta voluntad. Voluntad puede implicar querer y desear. En general, se trata de formas de la voluntad que van de la posibilidad real o cercana de acción de una emoción (querer) o formas lejanas o tendencias de la emoción (Mell, 1994, p. 71).
} 
Según Vargas, la formación se comprende como "la incidencia sobre la síntesis pasiva" (Vargas, 2016, p. 3). Esto quiere decir que los valores, sentidos y saberes que están en el sedimento pasivo pueden o han sido intervenidos por procesos activos. Como ya se ha descrito, la relación entre actividad y pasividad es mediada por percepciones y motivaciones: diferentes direcciones de la constitución (tanto subjetivas como de las cosas mismas) que expresan vínculos no causales entre estas formas de síntesis. Quiere decir que no hay proporción o causalidad directa, explicativa, determinadora, entre pasividad y actividad. Para el caso de la formación, la pregunta se dirige a las maneras en que la explicitación (la apercepción de la formación) orienta la comprensión de esta relación.

Necesariamente, esta explicitación es actividad. Está dirigida por el yo pienso (acerca de la formación para el caso) y el yo puedo pensar sobre la formación (en últimas: como de lo activo llego a pasividad). Es decir, desde el inicio hay una actividad que expresa reflexión sobre su actuar en la pasividad. Como un darse cuenta de (en este caso: dar cuenta de la propia pasividad). Ahora, en un segundo momento, la reflexión es sobre posibles índices de la relación comprensiva entre actividad y pasividad en la formación. Teniendo en cuenta que para la relación general tratada en la segunda parte de este escrito propone como base la motivación y la percepción, se postulan dos índices que pueden ser vías de comprensión y proyección de incidencias activas en la pasividad (en la formación): 1. Sensibilidad, como modo de la percepción, y 2. Voluntad, vinculado con la motivación.

\section{Sensibilidad [T3]}

Sensibilidad, para Husserl, se vincula con el nivel inferior de la constitución del mundo, el de la experiencia real de los cuerpos. "A él pertenecen todos los cuerpos individuales. Con la experiencia sensible se enlazan los sentimientos e impulsos sensibles" (Husserl, 2014, párr. 50, p. 243). Sensibilidad es propiedad perceptiva que permite llegar a los protoobjetos: los datos de sensación primitivos "que no están ya constituidos por ninguna actividad del yo, sino que son, en el sentido más estricto, predaciones para toda actuación del yo. Son 'subjetivos', pero no actos o estados del yo, sino tenencias que se imponen al yo" (Husserl, 2014, párr. 55, p. 262). Entonces, la sensibilidad de la sensación se presenta como actividad del yo que "presupone afección". Sin embargo, esta actividad no es solo receptividad y, en palabras de Husserl, "pasividad en el 
sentido del ceder" (Husserl, 2014, anexo XII, párr. 3, p. 390), sino una actividad de ceder libre, es decir, la sensibilidad es ubicación o posición con respecto a las afecciones.

Sensibilidad se puede asumir como percepción simple de cosas que trasciende a percepciones completas o series de percepciones. Para Husserl, esta percepción simple está relacionada con una síntesis particular denominada síntesis estética (2014, párr. 19, p. 9400). A diferencia de la síntesis categorial, en la estética se constituyen objetos desde las notas sensibles y que siempre remiten a síntesis continuas, es decir, en la cual la unidad está dada en los datos mismos y no en procesos categoriales. De esta forma, la sensibilidad implica actividad del yo y síntesis estética.

La actividad sensible tiene correlación cercana con pasividad en el sentido que el ceder libre de la sensibilidad requiere predaciones o esquemas de receptividad fundados pasivamente. Es la actividad motivada que dirige la mirada por las notas sensibles de las cosas y es el vínculo del yo trascendental con el yo personal. Este, cuya experiencia se orienta por el mundo natural, donde el yo se orienta por "su ser o su modo de estar hechas, por su belleza, su encanto, su utilidad, suscitan su interés, excitan su deseo de disfrutarlas, de jugar con ellas" (Husserl, 2014, párr. 50, p. 235). Por esta razón, la sensibilidad como actividad del yo tiene vínculo con la formación, más que la actividad predicativa. En la formación, la sensibilidad orienta los caminos y posibilidades de las demás actividades: funda marcos que direccionan el interés y los siembra con facilidad en pasividad.

Se puede considerar que, entre las relaciones entre actividad y pasividad, la sensibilidad implica una actividad fundante de la predación - la pasividad - Con esto mismo se puede considerar que la formación está llena de valores y sentidos ligados a la belleza, lo disfrutado, el gozo, el ocio. Cabe la duda

\section{Voluntad [T3]}

Voluntad es motivación subjetiva que expresa querer y deseo de movimiento (corporal). Es expresión contraria a la respuesta determinada psicofísicamente. Así, voluntad implica la actividad del yo anímico. Al igual que el causar psicofísico, la voluntad como causar psíquico puede explicarse, aunque Husserl no lo hace en Ideas II (cfr. Ideas II, § 59, p. 260). Describe solo que la voluntad opera como unidad del cuerpo: el sujeto para querer no requiere un saber fisiológico, sino un modo de actuación de lo anímico con el cuerpo que es unidad en el acto 
mismo. También afirma que lo contrario a la voluntad es el arbitrio (Ideas II, § 59, p. 306). Lo involuntario puede asumirse como lo automatizado: el movimiento que se hace sin querer.

Voluntad puede asumirse como un modo de la motivación, en este sentido, también ordena en la corriente de vivencias la constitución de las cosas. Con respecto a la formación, voluntad es actividad espiritual en la que se dispone toda la serie de intenciones. Estos modos de querer y deseo son expresiones de la relación pasividad-actividad en el sentido de que el acto voluntario les unifica en virtud de la satisfacción del yo personal. Es decir, es unificador vinculante del mundo real con la constitución trascendental del yo. Además, voluntad, como lo explica Mell (1997), también tiene formas activas y pasivas. Las activas como el querer, que tiene emoción por algo y a la vez posibilidad de acceso, y el deseo como voluntad a largo plazo (Según Mell, a esta voluntad Husserl la llama tendencia).

Este deseo (tendencia) es voluntad hecha pasividad. Con respecto a la formación se puede comprender la voluntad (deseo y querer) como componente que mueve síntesis activas a pasivas y viceversa. De actividad a pasividad dado que todo acto voluntario implica decisión manifestada; es decir, el acto voluntario parece contener de alguna forma querer y deseo (tendencia). De pasividad a actividad ya que siempre el acto voluntario retoma valores y sentidos en pasividad para poder actuar. Formación requiere de la voluntad: la pasividad toca los aspectos profundos del deseo. Queda por discutir si el deseo también es capturado por el sistema o siempre se puede evocar la libertad como propiedad inequívoca del sujeto para elegir.

\section{Cierre [T2]}

En la constitución pasiva hay intervención de la actividad. En este escrito se ha afirmado que esa intervención tiene relación con motivación y percepción. Trascendido el asunto a la formación, motivación se puede comprender como deseo, orientación particular decidida del yo; y percepción se desarrolla con el índice sensibilidad, que postula la síntesis estética como actividad primaria cercana a las síntesis pasivas. Sobre estas afirmaciones, se puede agregar para el asunto de la formación el de la ejercitación. Lo dado en pasividad se reaprende con práctica (ejercitación) y esta (que no necesariamente repetición) promueve lo que se sedimentará en pasividad. Afirma Husserl: 
Yo puedo tocar el piano. Pero no siempre me sale bien. Lo he desaprendido, he perdido práctica. Yo ejercito mi cuerpo. En las actividades más comunes generalmente no pierdo la práctica. Pero cuando he estado enfermo mucho tiempo acostado, luego tengo que volver a aprender a andar, y rápidamente vuelvo a hacerlo. Pero puedo también enfermarme de los nervios, pierdo el dominio sobre mis miembros, "no puedo". En este respecto me he vuelto otro. "Soy corporalprácticamente". (2014, párr. 59, p. 254).

La actividad es expresión del yo puedo, y además le ubica en contextos de posibilidad. Comprender los modos de la actividad es la forma de interpretar lo dado en pasividad. En este sentido, también esta comprensión de la actividad puede ubicar formas de captura de la pasividad, vinculadas a formas de ver y vivir en sociedad. Para los ejes relevantes presentados en este escrito, la intervención desde lo sensible (lo estético) y el deseo puede promover acceso a la formación comprendida como pasividad.

Para concluir con el ejemplo inicial: la primera relación, la tesis general, es que se aprendió a manejar bicicleta por conjunto de actividades. A su vez, a la pasividad ya establecida, en este caso saber manejar bicicleta, le corresponde una manifestación activa, que es el hecho fáctico de manejar bicicleta (yo puedo manejar bicicleta). Sin embargo, más que el conocimiento técnico, entre las actividades y las pasividades se propone tener en cuenta la sensibilidad: lo bonito de la bicicleta o el camino o lo placentero de hacerlo en compañía; y la voluntad: que de lo subjetivo lleva a desear-querer montar la bicicleta. Además, la voluntad-tendencia que hace y da sentido, a la práctica. 


\section{Referencias Bibliográficas [T2]}

Husserl, E. (2014). Ideas relativas a una fenomenología pura y una filosofía fenomenológica. Libro segundo: Investigaciones fenomenológicas sobre la constitución. México: UNAM, Instituto de Investigaciones Filosóficas, Fondo de Cultura Económica.

Melle, U. (1994). La fenomenología de la voluntad de Husserl (Trad. Carlos Eduardo Maldonado). Ideas y valores, (95), 65-84.

Merleau-Ponty, M. (1945/1975). Fenomenología de la percepción. Barcelona: Península.

Vargas, G. (2016). Programa de seminario: Pasividad y Formación en Ideas II de Edmund Husserl. Universidad Pedagógica Nacional. Documento del Doctorado Interinstitucional en Educación. 